\title{
La inversión en innovación tecnológica: la clave de américa latina frente a escenario global postcrisis
}

\section{Investment in technological innovation: the key of latin america against global scenario postcrisis}

Recibido: 23-04-2019 / Revisado: 13-05-2019 /Aceptado: 14-06-2019/ Publicado: 05-07-2019

\author{
Enma Elizabeth Curillo Gonzalez. ${ }^{1}$, Wendy Nathaly Espinoza Espinoza. ${ }^{2} \&$ Flor María Lorena \\ Estrada Carrera. ${ }^{3}$
}

\begin{abstract}
DOI: https://doi.org/10.33262/cienciadigital.v3i3.632

This article exposes the origin of the global economic crisis of 2008, and how it, in turn, reconfigured the globalization and the international trade. The genesis of the crisis is established, its repercussion in a new fragmentation of the global production chain whose value is generated through investment in technological innovation. It highlights how the weak institutionality of the international economy does not contribute to: (a) reducing the financial and technological gap between industrialized and peripheral countries, such as the Latin American nations, (b) nor helping to dismantle the protectionist barriers of the economies strong, and (c) much less helps to cope with the global trend to limit foreign financing. Faced with this complex and competitive scenario -where the competition and influence of the BRICS- is present, it is explained how Latin America has no other option than to invest in technological innovation to overcome the structural heterogeneity of its countries' production, incorporate value in the global productive chains and conquer or create market niches. For this purpose, it is necessary to work in concert in the regional blocks to establish joint strategies in the international trade scenario.
\end{abstract}

Key words: Technological Innovation, International Trade, Globalization, Post-Crisis Global Scenario, Latin America.

\section{Resumen.}

En este artículo se expone el origen de la crisis económica mundial de 2008, y cómo esta a su vez, reconfiguró la globalización y el comercio internacional. Se establece la génesis de la crisis, su repercusión en una nueva de fragmentación de la cadena de producción mundial en cuyo trayecto se genera valor mediante la inversión en innovación tecnológica. Se destaca cómo la débil institucionalidad de la economía internacional no contribuye a: (a)

\footnotetext{
${ }^{1}$ Consejo de la Judicatura Esmeraldas, Ecuador, enma.curillo@funcionjudicial.gob.ec

${ }^{2}$ Universidad de Guayaquil, Ecuador, wendy.espinozaes@ug.edu.ec

${ }^{3}$ Universidad de Guayaquil, Ecuador, flor.estradac@ug.edu.ec
} 
reducir la brecha financiera y tecnológica entre países industrializados y periféricos, tal como lo son las naciones latinoamericanas, (b) ni ayuda a desmontar las barreras proteccionistas de las economías fuertes, y (c) mucho menos coadyuva a enfrentar la tendencia mundial a limitar el financiamiento extranjero. Ante este escenario complejo y competitivo -donde adicionalmente está presente la competencia e influencia de los BRICSse explica cómo a América Latina no le queda otra opción que invertir en innovación tecnológica para superar la heterogeneidad estructural de la producción de sus países, incorporar valor en las cadenas productivas mundiales y conquistar o crear nichos de mercado. Para tal efecto, es menester trabajar de forma concertada en los bloques regionales para establecer estrategias conjuntas frente el escenario del comercio internacional.

Palabras clave: Innovación Tecnológica, Comercio Internacional, Globalización, Escenario Global Postcrisis, América Latina.

\section{Introducción.}

\section{La crisis de 2008: el inicio del cambio del escenario económico global}

La crisis financiera internacional de 2008 se originó a causa de los problemas generados por las hipotecas subprime de Estados Unidos y se extendió luego a otros sectores de la economía, conmoviendo no sólo al mercado de esa nación, sino también a las de otros países del orbe, de forma más significativa a la eurozona. Como es común en estas situaciones, la contracción económica y el aumento del desempleo ha tenido un mayor impacto en comparación a lo acontecido en el país de origen. Cabe destacar, que en los inicios de la crisis no tuvo mayor repercusión en las economías emergentes, sin embargo, las secuelas financieras las alcanzaron.

Esta crisis, la mayor desde la Gran Depresión de 1929, ha orientado el desarrollo de la economía global; por ejemplo, luego de la debacle, en los cinco años posteriores hubo un decrecimiento de esta seis puntos porcentuales en términos reales que si lo hubiera hecho a la tasa promedio de los veintiocho años previos: durante 1980-2007, la economía del orbe creció a 3\% promedio anual, mientras que a partir de 2008 y hasta 2012, lo hizo a 1.8\%, según cifras del Fondo Monetario Internacional (FMI, 2012).

Cabe destacar que en la primera década del siglo XXI, el desarrollo económico de Estados Unidos representaba un déficit considerable de cuenta corriente, el cual, como porcentaje del PIB, alcanzó $6 \%$ en 2006. En un contexto frenético de tasas de interés bajas y cuantiosa liquidez, propendido por la política monetaria estadounidense, genero déficits que se financiaban sin dificultad con entradas de capital. Simultáneamente, sucedía en el orbe, y en específico, en Estados Unidos, un proceso de la innovación financiera sustentada en la expansión del crédito; el cual tenía por objeto aumentar la rentabilidad de los capitales financieros que se ofrecían con facilidad por la banca. La innovación financiera, a su vez, permitía mayor disponibilidad de recursos para los agentes económicos.

En retrospectiva, se avizora que las autoridades monetarias y financieras de Estados Unidos no percibían al déficit de cuenta corriente como un problema: la productividad crecía y las empresas 
reportaban aumento de las utilidades, sobre todo las del sector financiero, estos dos factores se incidieron para que se asumiera el déficit de cuenta corriente como un efecto del equilibrio. La lógica era que a mayor ingresos de capital por mayor productividad y utilidades de las empresas debían generar déficit de cuenta corriente como respuesta. Se partió del supuesto que una economía grande y fuerte como la de Estados Unidos, con el sistema financiero más fluido y avanzado del mundo, podía arreglar o solucionar fácil ese déficit, que nunca se detuvo.

A contracorriente de este exceso de confianza, la expansión acelerada del crédito y la innovación financiera tenían un manejo turbio omitido por las autoridades financieras y monetarias estadounidense: crecía debido a una débil regulación ante las prácticas, tales como otorgar créditos y financiamientos sin un respaldo demostrable del solicitante. Las instituciones financieras, con operaciones no reguladas, se propagaron de manera exponencial porque justo allí residían las grandes ganancias. Las mismas instituciones bancarias y crediticias sujetas a regulación buscaron la forma de establecer otras instituciones fuera de la regulación bancaria (Rodríguez y Zurita, 2008).

La inequitativa información, común en el sistema financiero, produjo que muchos inversionistas no se percataran de los riesgos de activos respaldados por productos surgidos de la innovación financiera. Invertían sin preocuparse por los respaldos y se concentraban en la rentabilidad del crédito fácil y bursatilización rápida. Prevaleció el riesgo, el exceso de confianza y se decidió omitir la regulación bancaria y el respaldo para otorgar créditos, considerados como ortodoxos y hoy se reconocen como necesarios: se creó un escenario próximo a la corrupción.

Se deformaron los estímulos que generaron el riesgo y descartaron la actuación prudente y moral, sobre todo en la protección de los activos de las instituciones financieras. Con la bursatilización instantánea de la cartera de crédito, se vendieron despreocupadamente sus derivados, con ello descartaron el riesgo crediticio y, supuestamente, sorteaban pérdidas en el mediano y largo plazo.

En este contexto era posible bursatilizar hipotecas de alto riesgo con pocas preocupaciones ni procesos burocráticos. Se encubría el alto riesgo en los derivados financieros y se les transferían a otros agentes económicos, usualmente, a otras instituciones financieras, actitud que se viralizó. Se asumió como negocio abrir un gran número de hipotecas, bursatilizarlas y evitar el riesgo, casi con total autonomía de la capacidad de pago de los acreditados. De esta forma, las instituciones financieras emprendieron operaciones de crédito a corto plazo con pasivos excluidos de sus balances (CEPAL, 2009). Esto abrió una ingente fragilidad del sistema financiero de Estados Unidos y de la economía global.

La crisis se originó cuando decrecieron los precios de los bienes raíces. Cuando los adeudados presentaron morosidad con los pagos de sus hipotecas, en su mayoría, de baja calidad crediticia (subprime), este frágil y casi falso sistema financiero eclosionó (Lane, 2013). La desesperación se hizo viral. Como no se conocía la dimensión de los pasivos netos fuera del balance de las instituciones financieras, se detuvo el crédito, y esto incitó la crisis sistémica (CEPAL, 2009). 
La crisis que parecía un asunto doméstico de Estados Unidos, se extendió rápido a otras regiones del globo (Chinn, 2012), especialmente, a otras economías avanzadas como la Eurozona, Japón, Australia y Nueva Zelanda, así como hacia las emergentes (Eichengreen, 2012). Como medida para contrarrestar esta crisis, paradójicamente, estos países de economía avanzada aplicaron políticas monetaria de respaldo y auxilio a las instituciones financieras, medidas que se supone son propias de los países tercermundistas, consistentes en otorgar facilidades de crédito público y crédito en cantidades extraordinarias a las instituciones financieras para evitar una crisis de liquidez y ulterior estanco de la economía (Nayak, 2013; Tanzi, 2011). En general, el proceso de transmisión de la crisis financiera de Estados Unidos hacia otros países del mundo acaeció mediante el propio sector financiero como del sector real.

Efectos de la crisis de 2008 en la economía mundial

La crisis no ha afectado, máxime ha acelerado el proceso de globalización, especialmente en tres factores para la inserción de América Latina y el Caribe en el mundo: (a) la profundización y desarrollo del cambio tecnológico; (b) la presencia e influencia de China y otras economías emergentes en la economía mundial y (c) el desafío del cambio climático.

La aparición y desarrollo de la crisis puso en la palestra relieve la urgencia de explorar y definir reformas en la regulación financiera y en el funcionamiento del propio sistema financiero internacional. Estos retos se plantean de forma imperiosa en un escenario internacional con menor crecimiento que las últimas décadas del siglo XX y de los años anteriores a la crisis de 2008 (Kelly, 2006), particularmente en las economías industrializadas, urgidas de significativos ajustes en sus desbalances presupuestarios. Tanto las condiciones actuales como el temor generado por la crisis de 2008 crean un caldo de cultivo para el resurgimiento de medidas proteccionistas tanto de las economías desarrolladas, como las emergentes, hecho, que seguramente influirá en las decisiones en política comercial de los países latinoamericanos (Fitzgerald, 2007).

\section{Vulnerabilidades en la dimensión macroeconómica del mundo}

En el futuro inmediato, el comportamiento de la economía mundial será muy inferior al del período 2003-2007. Las causas de tal hecho son (Rosales, 2009):

Las 34 economías de la Organización para la Cooperación y el Desarrollo Económicos, OCDE, verán reducir su crecimiento potencial e incluso crecerían bajo ese ritmo por causa de una menor tasa de inversión y un mayor nivel de desempleo.

El comercio mundial creció a tasas inferiores a las demostradas en el ciclo expansivo 2003-2007. Pero, entretanto, el comercio de los países industrializados crece poco, los intercambios comerciales internacionales de China y Asia se comportan más fluidas, sin embargo, no remontarán las cifras alcanzadas por la bonanza del lapso previo a la crisis. - Una tendencia a mantener políticas proteccionistas como consecuencia de la crisis, sin que se posible desmontarlas y sustituirlas por otras más viables, sanas y sostenibles, una vez que se equilibre y recupere la economía mundial. 
Una capacidad excedida de varios sectores productivos, que, a su vez, genera una demanda internacional limitada, esto traerá como consecuencia una mayor competitividad en el sector de oferta.

Durante el 2010-2011 la economía mundial recuperó crecimientos anuales cercanos al 4\%. Pero el contraste e interesante de este escenario es que las economías industrializadas crecieron alrededor de $2 \%$ contra un $6 \%$ de las economías emergentes, y en especial de los BRIC's.

La heterogeneidad en la producción también se está dando en las economías en desarrollo pues mientras las economías asiáticas están próximas a un crecimiento de $8 \%$ en América Latina apenas se llega a la mitad de ese desarrollo:

Según se ha mostrado en diversos estudios, el desarrollo industrial de América Latina ha estado marcado desde sus inicios por la coexistencia de un sector exportador y de algunas actividades manufactureras de elevada productividad, en ciertos casos comparable con la de las economías desarrolladas y de una agricultura no exportadora y servicios, en particular los correspondientes al sector informal, de muy baja productividad (Capdevielle, 2005: 102).

Por su parte, la recuperación en las economías industrializadas, además de ligera, sigue dependiendo temerariamente de las medidas fiscales, monetarias y financieras aplicadas para combatir la crisis, es decir, inyecciones de capitales desde el sector estatal. Se corre el riesgo que ante una poca demanda privada, los mercados financieros vuelvan a activarse, con poca correspondencia del mercado real. Se vuelve a la creencia de la ganancia fácil y la bursatilización rápida, sin que se hayan cumplido con las medidas financieras planteadas a fines de 2008. Aumentan las expectativas sobre la sostenibilidad fiscal en las economías industrializadas, con niveles de déficit fiscal de dos dígitos, como porcentaje del PIB, y con deuda pública en aumento (Rodríguez y Zurita, 2008).

Las restricciones fiscales y financieras en la OCDE coartarán el crecimiento económico. Estas medidas apuntan que resta aún un margen amplio de arreglo en las carteras de las familias y de las instituciones financieras en las economías industrializadas, específicamente en Estados Unidos, para ajustar sus balances, restringir los niveles de endeudamiento (OCDE, 2008).

En consecuencia, se abre un escenario más bien restrictivo de la demanda por importaciones en esas economías, siendo idóneo que la economía mundial halle nuevos resortes e incentivos de demanda. China y las demás economías emergentes aún no hacen contrapeso a las economías industrializadas en el crecimiento de la demanda global y como mercados para las exportaciones mundiales (Kelly, 2006). Aunque el peso relativo de las economías emergentes en la economía ha crecido y sigue en aumento, esto no es suficiente para compensar el desaceleramiento económico de Estados Unidos y la Unión Europea (Fitzgerald, 2007). En todo caso, este reacomodo del comercio mundial pone de relieve la importancia creciente del comercio Sur-Sur. 
De este modo, la economías industrializadas, enfrentarán retos importantes como (Rosales, 2009): (a) decidir cuándo y cómo retirarán completamente las ayudas fiscales, monetarios y financieros, sin afectar la 'débil recuperación; (b) la restauración de los balances financieros de familias y entidades financieras, particularmente en Estados Unidos; (c) el control y la reducción gradual del déficit fiscal y la deuda pública; (d) el restablecimiento de controles regulatorios a la banca y las finanzas que impidan la repetición de crisis tan profundas como la del 2008.

Estas medidas se han de tomar en un escenario de limitado crecimiento, con tasas de desempleo cercanas al 10\% en Europa y Estados Unidos y con la subsistencia de varias de las políticas proteccionistas -comerciales y financieras-aplicadas durante la crisis. En los próximos años, habrá menos disponibilidad de financiamiento internacional. La fuerte disminución de la riqueza acumulada en todo el mundo, principalmente en los países industrializados, cambió el panorama de liquidez virtualmente infinita y con bajo riesgo por otro de liquidez acotada y riesgos variables. El vínculo entre los flujos financieros y los productivos reales, así como de estos con el comercio internacional tendrán que reorientarse (Hamel, 2008).

En consecuencia, una menor liquidez mundial llevará a una reducción de los flujos de capital hacia América Latina y el Caribe y las otras regiones en desarrollo (Rosales, 2009). Además, las elevadas necesidades de financiamiento de los gobiernos de la OCDE competirán con las de los países en desarrollo y de las economías emergentes (OCDE, 2008). Es probable que algunos tópico novedosos, como los fondos soberanos de las economías emergentes y la internacionalización de las empresas chinas, pasen incursionar de forma más significativa en el nuevo orden de las finanzas internacionales dada, por una parte, la gran liquidez que han acumulado las empresas y los bancos chinos y las economías petroleras, y por la otra, la baja rentabilidad que estarían entregando los papeles del tesoro de los Estados Unidos y otros países industrializados (Fitzgerald, 2007).

2. Problemática de las instituciones del comercio internacional

La crisis 2008 puso al descubierto la debilidad institucionalidad del comercio y su poca pertinencia con los nuevos desafíos del siglo XIX. El G-7, conformado por las economías más fuertes del mundo: Estados Unidos, Canadá, Alemania, Francia, Italia, Reino Unido y Japón, fue probablemente uno de los actores más afectados por la crisis, al quedar en evidencia que todos sus integrantes eran vulnerables en conjunto por un error de uno de sus miembros, y dejó claro que una vez superada la recesión internacional obligaría a incluir en la mesa de negociaciones a otros países, entre ellos las economías emergentes (Fitzgerald, 2007).

La redimensión del G-7 al G-20 refleja el nuevo orden económico mundial al cual ha de ajustarse América Latina, suponiendo una mayor competitividad tanto para créditos como para las exportaciones. El grupo se amplió a: Alemania, Arabia Saudita, Argentina, Australia, Brasil, Canadá, China, Corea del Sur, Estados Unidos, Francia, India, Indonesia, Italia, Japón, México, Reino Unido, Rusia, Sudáfrica, Turquía, y una representación adicional por la Unión Europea.

La tardanza de respuesta del FMI y del Banco Mundial a la crisis de 2008, así como su incapacidad para anticiparla, ha dejado en evidencia la necesidad de reformar y fortalecer a tales organizaciones 
multilaterales, de tal forma que sea vuelva eficiente y optimice la regulación financiera de los países que la conforman. Adicionalmente, el fracaso de las metas de la Cumbre de Copenhague sobre cambio climático devela el rezago de estas instituciones frente al avance de la globalización y la innovación tecnológica del comercio internacional (Friedman, 2006).

Es un asunto de gobernanza de la globalización económica, comercial y financiera, que puede enunciarse fácilmente, pero es difícil y complejo de cumplir, sino que los retos son muchos más: monitoreo, control y prevención de pandemias, flujos migratorios, cambio climático, narcoterrorismo, estados fallidos, guerras civiles, conflictos regionales, preservación del medio ambiente.

\section{La innovación y del cambio tecnológico: la clave de la economía global y el comercio internacional actual}

El impacto de la innovación tecnológica actual está perfilando los escenarios competitivos de un sinnúmero de sectores productivos. Esta vertiginoso cambio tecnológico radica en una variedad de tecnologías de la información y de las comunicaciones que de forma orgánica están redibujando la economía global y, por ende, el comercio internacional (Kelly, 2006). El espectro integra: la biotecnología, nanotecnología, desarrollo de nuevos materiales y fuentes energéticas renovables (Rosales, 2009).

Estas tecnologías son transdisciplinarias y están transformando completamente la producción y los servicios, induciendo innovaciones de procesos, productos y modelos de negocios (Friedman, 2006; Rosales, 2009). Los estados que se adapten y respondan a estos desafíos, posibilidades y oportunidades de esta innovación tecnológica podrán tener un mejor desenvolvimiento en la globalización y una presencia más activa en el comercio internacional (Hamel, 2008). Pero es justo en este terreno donde los países de América Latina, tanto del sector público como privado tienen más resistencias: no invierten en los intangibles, verbigracia, la innovación y la tecnología.

La innovación tecnológica en los procesos productivos cada vez es más intrínseca o biunívoca con la competitividad. El poco acceso al financiamiento internacional incide negativamente en las inversiones a largo plazo (energía y minería), o en los sectores que necesitan un alto nivel de inversión para ser competitivos desde el campo tecnológico (manafactura).

La debacle financiera dejó a sectores productivos con exceso de capacidad, que tiene como contraparte una demanda escasa, lo que lleva a la conformación de oligopolios mediante el proceso de fusiones y adquisiciones de empresas. Adicionalmente, la pérdida de valor de activos ha ocasionado la capitalización fácil de quienes tenían capacidad de compras. La etapa posterior a la crisis tiene una tendencia a un mayor nivel de concentración económica y destacará la importancia de las políticas de competencia en las economías nacionales y en el comercio internacional (Hamel, 2008).

Es perentorio renovar los modelos de negocios productivos y de comercio exterior basados en la innovación tecnológica. En un escenario global con un comercio internacional ralentizado y limitado financiamiento externo, altamente competitivo y políticas proteccionistas de los estados 
nacionales, la reacción previsible de las empresas es: reducir costos, posponer inversiones, medidas inmediatistas, y defensa de sus principales mercados y clientes (Rosales, 2009). Sin embargo, esto necesariamente debe ir acompañado de una reingeniería del modelo de negocios a largo plazo. A este respecto cabe destacar:

En la visión de Prebisch, la importancia de las asimetrías que caracterizan el sistema económico internacional implicaba, en cualquier caso, el reconocimiento de que la responsabilidad en el desarrollo descansa, en última instancia, sobre los países en desarrollo. La corrección de estas asimetrías contribuía a construir un "ambiente facilitador" para los esfuerzos por generar un "desarrollo desde dentro" (Ocampo, 2001: 8).

Es imperioso descubrir y aprovechar las nuevas oportunidades mediante una adecuada innovación tecnológica tanto de los procesos productivos como el diseño organizacional, que contribuyan a develar las ventajas competitivas de las empresas, para lo cual sin duda es imprescindible un avezado conocimiento mercadológico. La prospectiva para configuración de un nuevo portafolio de negocios es más fructífero que deslocalizar la cadena de producción, más que esta acción, se trata de analizar cómo las cadenas globales y regionales de valor se redefinen de acuerdo con (Rosales, 2009): (a) la capacidad de recuperación de las diversas economías frente a la crisis, (b) el acceso a materias primas escasas, (c) revalorización de ventajas geográficas o logísticas (ventajas comparativas), como por ejemplo, la proximidad a los principales centros de consumo, o de la presencia de talento en disciplinas tecnológicas determinadas.

Es menester trascender los retos de la globalización antes de la crisis de 2008, pues hay desafíos nuevos cuya única respuesta está en la investigación tecnológica emparentada con estrategias de marketing para acceder o crear nuevos mercados allende a las fronteras, crear valor en las cadenas de producción mediante innovación, y la capacidad de reingeniería para adaptarse a estos cambios en un mundo con restringidos financiamientos, alta competitividad y estados recelosos y proteccionistas.

Se está asistiendo a una marcada tendencia a establecer y desarrollar la producción mediante a cadenas globales de valor. Esto alude a la segmentación geográfica de los procesos productivos, a través del beneficio de la digitalización de las tareas, una creciente internacionalización y comercialización de los servicios y la disminución de los costos de transporte y logística. Las empresas marchan en el marco de una cadena de producción mundial y funcionan como una unidad, ya que, se comunican gracias a internet. Los progresos tecnológicos traen aparejados rediseños de la organización de la producción que replantea lo geográfico.

Ha surgido la organización extendida, es decir el traslado de una parte de la producción al exterior con control estricto de la empresa matriz, de allí que cada vez más la producción, la innovación, el marketing y el comercio internacional son procesos simultáneos e interdependientes. (Hamel, 2008) Otras formas de organización no tan recientes son el outsourcing o el insourcing. Todo este escenario es posible gracias a las políticas librecambistas y la apertura gradual de los mercados 
nacionales al comercio y las inversiones, mediante la cesación de aranceles (Kelly, 2006), aunque aún haya medidas proteccionistas afianzadas por la crisis de 2008 que mantienen aranceles preferenciales en los bloques regionales.

La progresiva integración de las economías en este mercado global manifiesta un escenario diverso de oportunidades y amenazas para empresas, sectores, países y regiones (Kelly, 2006). De acuerdo con Bekerman y Sirilin (1995: 66): "el conocimiento y el avance científico técnico son factores que determinan el desarrollo de nuevas ventajas comparativas".

Es notorio que el desarrollo de los países dependerá cada vez más de la capacidad de moverse en un escenario donde la inversión, el marketing, la producción, la rentabilidad están transversalizadas y relacionadas por la innovación tecnológica. En efecto, la innovación y las nuevas tecnologías brindan nuevas oportunidades para recortar la brecha con los países industrializados, si sabe esquivar las medidas proteccionistas, sin dejar de incentivar, al menos temporalmente aquellos sectores donde se sea competitivo a nivel mundial, o se posea ventajas comparativas o competitivas para atender o crear mercados en el extranjero (nichos de mercado). Esta es la estrategia que deben implementar los países de América Latina. En este contexto, se subraya:

En países con mercados pequeños (sin empresas lo suficientemente grandes como para alterar las reglas de la competencia estratégica internacional), aún existe la posibilidad de aplicar políticas comerciales en aquellos sectores con rendimientos normales que utilizan economías de escala indirectas vinculadas, por ejemplo, al transporte y a la comercialización internacional (Bekerman y Sirilin, 1995: 73).

Lo anterior supone un inmenso esfuerzo en el interior de los países latinoamericanos para fortalecer el talento humano y la configuración de redes internacionales de innovación y negocios tecnológicos, asociando la estructura productiva y exportadora con investigaciones en innovación y conocimiento; sobre todo las relacionadas a la nanotecnología, biotecnología, nuevos materiales, energías sostenibles, informática, telemática, reingeniería organizacional. Todo esto incorporando la prospectiva y el marketing que permitan identificar posibles nichos de mercado a partir de ventajas comparativas y competitivas de empresas, sectores o países.

Tal reto es posible si se incorpora la innovación tecnológica a las actividades productivas, para lograr mayor productividad, trascender y superar la heterogeneidad estructural (reduciendo así las brechas internas de productividad e ingresos) propia de los países latinoamericanos. En este sentido, cabe destacar:

tal desigualdad estaba determinada por las diferencias tecnológicas existentes entre un grupo reducido de actividades productivas, cuyas prácticas se hallaban próximas a la frontera del conocimiento, y un grupo más numeroso de actividades, en que predominaban condiciones productivas y tecnológicas atrasadas, que empleaban en forma ineficiente los factores productivos y pagaban bajas remuneraciones (Capdevielle, 2005: 101). 
La competitividad es sistémica, pues la innovación productiva debe contener todo el contexto donde se inscriben las empresas: la infraestructura, la tecnología, energía y transporte; el sistema educativo; las relaciones entre empleadores y empleados; el aparato institucional público y privado y la red financiera. La impronta sistémica de la competitividad requiere beneficiar la integración del sistema productivo y la homogeneización gradual de los niveles de productividad de los sectores y al interior de ellos: "la heterogeneidad estructural refleja la incapacidad de un modelo de desarrollo dado de difundir las prácticas productivas y tecnológicas óptimas, hacer un uso eficiente de los factores y beneficiar a sus propietarios con una retribución adecuada" (Capdevielle 2005: 101).

La colaboración e integración regional es fundamental para optimizar la competitividad, ya que los retos de la nueva economía mundial trascienden las capacidades nacionales. Es necesario aprovechar los bloques de integración latinoamericanos para ir de forma concertada y estratégica frente a los foros internacionales donde no solo escaso financiamiento (OCDE, 2008), sino la competitividad tradicional del G-20 más la competencia reciente de los BRIC's.

De hecho, una óptima contribución permitiría congeniar y armonizar los esfuerzos para obtener los niveles de calidad, escala y productividad requeridos en la economía global. Es necesario que los bloques regionales abran espacios para la formación, captación, intercambio y aprovechamiento del talento, artífice de la innovación tecnológica creadora de valor en las cadenas de producción mundiales. Así mismo, los países latinoamericanos eventualmente se beneficiarían de los bloques de integración regionales para intercambiar experiencias sobre iniciativas exitosas de innovación, competitividad y concertación de políticas entre actores públicos y privados.

\section{Conclusiones}

La crisis financiera de 2008 trajo un cambio radical en la economía del mundo. El exceso de confianza de la economía robusta de Estados Unidos llevó al sector financiero de ese país a aplicar estrategias de bursatilización rápida de créditos, muy próximos a la corrupción, hecho que fue posible por la omisión de las regulaciones en el otorgamiento de créditos. Este desliz, junto a otros factores, creó una burbuja financiera que llevó a una crisis económica mundial resultando la Eurozona, Japón, Australia y Nueva Zelanda afectados, y en menor medida, los BRIC's y los países de la periferia. Tal situación ha creado el retorno de medidas proteccionistas y de inyección de capital al sector bancario por parte del estado en los países industrializados, reduciendo así el financiamiento internacional.

La crisis financiera de 2008 también dejó al descubierto la fragilidad de la institucionalidad financiera internacional, sobre todo el FMI y el Banco Mundial quienes no pudieron prevenir la crisis y no reaccionaron de forma acertada ante esta. Incluso, a más de diez años de la crisis económica mundial, aún la bursatilización inmediata de créditos e instrumentos financieros sigue siendo una tentación y opción para muchos inversionistas y negociantes de los países industrializados. La resulta de todo este escenario es una reducción del crédito o financiamiento exterior para América Latina. 
Para la región latinoamericana se suman los desafíos previos a la crisis de 2008 y posterior a ella, entre las que destacan la competitividad tradicional del G-20 (OCDE, 2008), la nueva competitividad y posicionamiento de los BRIC's, menor financiamiento exterior, medidas proteccionistas de las grandes economías, e introducción de la innovación tecnológica en las cadenas de producción mundial.

Este escenario no les deja otro camino a los países de América Latina que superar la heterogeneidad estructural, mediante la inversión de investigación e innovación tecnológica de los procesos productivos, los diseños organizacionales, la mercadotecnia, para identificar o crear nichos de mercados donde se pueda ser competitivo en el concierto del comercio internacional. La innovación tecnológica ha de servir para crear nuevos mercados, conquistar otros o competir desde ventajas comparativas añadiendo valor a las cadenas de producción mundial; lo cual es solo posible mediante investigaciones en el área de la biotecnología, nuevos materiales, nanotecnología, informática, telemática, energías ecológicas y optimación del sector manufacturero, entre otros.

Es la hora de buscar o crear oportunidades mediante la captación y aprovechamiento del talento que logre usar la innovación tecnológica como eje transversal de la producción, la reingeniería organizacional, el diseño de portafolios de negocios, la creación u ocupación de nichos de mercado (marketing); es decir, el comercio internacional definitivamente dejó de ser una actividad posterior y que surgía como una fase avanzada de las empresas cuando ya tenía excedentes o habían cubierto las demandas del mercado nacional; al contrario, el comercio internacional hoy día es una actividad simultánea con la producción y el marketing. La innovación tecnológica es la clave para realizar esta compleja y urgente sinergia, y por ende, necesita inversión tanto pública para los sectores potencialmente competitivos y por parte de los accionistas que no están consciente de la importancia que ha adquirido la innovación y desarrollo tecnológico en la actual fase de globalización.

Para lograr tal cometido es importante que en los bloques regionales de integración de América Latina, la inversión en innovación y la captación de talento humano sean temas de agenda y de concertación para poder incursionar exitosamente en una economía mundial más competitiva y con menor flujo de financiamientos. Es hora de ser estrategas desde la empresa, los sectores, los países y los bloques regionales de integración de América Latina.

\section{Referencias Bibliográficas}

Bekerman, M.; Sirlin, P (1995). Política comercial e inserción internacional. Una perspectiva latinoamericana. Revista de la Cepal, (55), 65-77. Disponible: https://repositorio.cepal.org/bitstream/handle/11362/11974/055065078_es.pdf?sequence=1\& isAllowed $=\mathrm{y}$

Capdevielle, M. (2005). Globalización, especialización y heterogeneidad estructural en México. En Heterogeneidad estructural, asimetrías tecnológicas y crecimiento en América Latina 
(Cimoli,M, editor). Organización de las Naciones Unidas: Santiago de Chile, pp. 121-126. Disponible:

https://repositorio.cepal.org/bitstream/handle/11362/2804/S2005051_es.pdf?sequence=1\&is Allowed=y.

CEPAL (2009). Panorama de la inserción internacional de América Latina y el Caribe 20082009. Crisis y espacios de cooperación regional. Disponible: https://repositorio.cepal.org/handle/11362/1172

Chinn, M.D. (2012). Chinn. Evidence on Financial Globalization and Crises: Global Imbalances. Encyclopedia of Financial Globalization. Elsevier.

Fitzgerald, E. (2007), Los nuevos colosos emergentes y su efecto sobre la economía internacional, Claves de la economía mundial, (7), Madrid, Instituto Español de Comercio Exterior.

FMI (2012). Base de datos del Panorama Económico Mundial. Estados Unidos.

Friedman, T. (2006), La tierra es plana. Breve historia del mundo globalizado del siglo XXI, Madrid, Martínez Roca.

Hamel, G. (2008), El futuro de la administración, Bogotá, D.C., Grupo Editorial Norma.

Kelly, E. (2006), La década decisiva. Tres escenarios para el futuro del mundo, Bogotá, D.C., Grupo Editorial Norma.

Lane, P. (2013). Financial Globalisation and the Crisis. Open Economy Review; 24 (3): 555p. Disponible: https://link.springer.com/article/10.1007/s11079-012-9266-0

Nayak, S. (2013). The Global Financial Crisis: Genesis. Policy Response and Road Ahead. Suiza: Springer.

Ocampo, J.(2001). Raúl Prebish y la Agenda del Desarrollo en los Albores del siglo XXI. En Seminario La teoría del desarrollo en los albores del siglo XXI. Santiago de Chile, 28 y 29 de agosto.Comisión Económica para América Latina. Disponible: https://repositorio.cepal.org/bitstream/handle/11362/21410/S33098S471RP2001_es.pdf?sequ ence $=1 \&$ is Allowed $=\mathrm{y}$

Rodríguez, J. y Zurita, J. (2008). La crisis de los créditos hipotecarios en Estados Unidos: algunas consideraciones. Análisis Económico; 23:331. Disponible:

www.redalyc.org/html/413/41311483016/ 
OCDE (Organización de Cooperación y Desarrollo Económicos) (2008), Staying Competitive in the Global Economy: Compendium of Studies on Global Value Chains, París.

Rosales, O. (2009). La globalización y los nuevos escenarios del comercio internacional. Revista Cepal, 97, pp. 77-95. Disponible:

https://repositorio.cepal.org/bitstream/handle/11362/11272/097077095_es.pdf?sequence=1\& isAllowed=y

Tanzi, 2011 Tanzi, V. (2011). La crisis financiera y económica de 2008-2009: Efectos fiscales y monetarios. Disponible en: http://www10.iadb.org/intal/intalcdi/PE/2011/08050.pdf

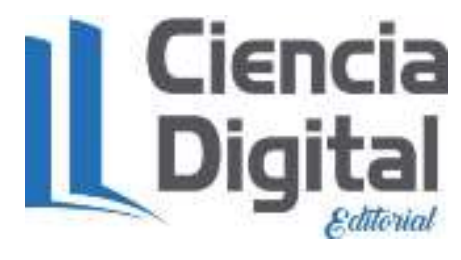


PARA CITAR EL ARTÍCULO INDEXADO.

Curillo Gonzalez, E., Espinoza Espinoza, W., \& Estrada Carrera, F. M. (2019). La inversión en innovación tecnológica: la clave de américa latina frente a escenario global postcrisis. Ciencia Digital, 3(3), 253-266. https://doi.org/10.33262/cienciadigital.v3i3.632

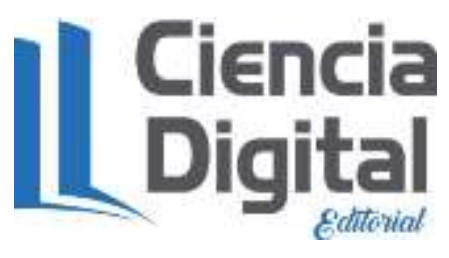

El artículo que se publica es de exclusiva responsabilidad de los autores y no necesariamente reflejan el pensamiento de la Revista Ciencia Digital.

El artículo queda en propiedad de la revista y, por tanto, su publicación parcial y/o total en otro medio tiene que ser autorizado por el director de la Revista Ciencia Digital.
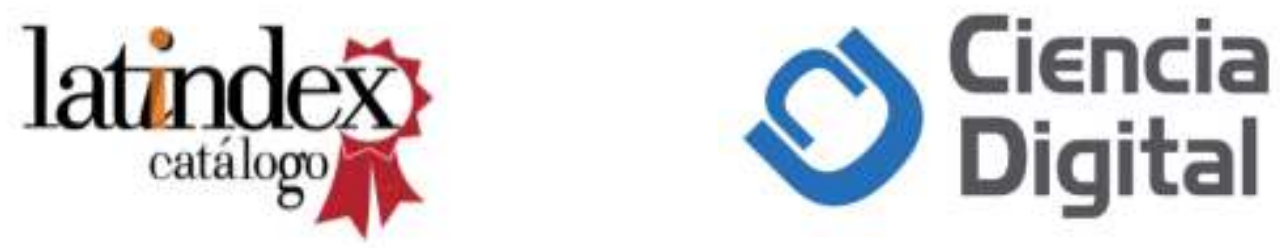